\title{
The Study of Heterographs in Chinese Northeast Dialect ${ }^{*}$
}

\author{
JIN Ya-shu \\ Yanbian University, Yanji, China
}

\begin{abstract}
Mr. Yin Huanxian is the first one who wrote article "Discussion on the Criterion of Words' Written Forms" in 1962. After the publishing of the article, Gao Gengsheng proposed the concept of "heterographs". For decades, a great many articles are published and ardent discussions involved in are holden. These articles mainly refer to the subject character of sorting out heterographs, the definition of heterographs, the classifying of heterographs as well as the way and principles of heterographs' criterion. Chinese Northeast dialect is one of the basic dialects in mandarin. With the development of the society, the influence of Northeast dialect is expanding. Because of its humorous and straightforward features, Chinese Northeast dialect was recognized and loved by more and more people. However, a large number of heterographs are produced in the formation of Northeast dialect. After inspecting, they are divided into three conditions on the whole, including homophones, dialectal heterographs and Yixu Heterographs, which are in the same word element but different order in it. Based on the concept and connotation, this paper focuses on studying, collecting and summarizing the "heterographs" in Chinese Northeast dialect, and describing the meaning of the standardization research of heterographs in Chinese Northeast dialect.
\end{abstract}

Keywords: heterographs, Northeast dialect, conditions, factors

\section{The Definition of Heterographs}

Heterographs phenomenon almost exists in every kind of language, so it reveals the universality of language. Besides, Chinese is a kind of language whose form indicates meaning. The form plays an important role in expressing concept and meaning, so the Heterographs phenomenon is more obvious than other languages.

It has been nearly a half century since Mr. Yin Huanxian wrote the article "Discussion on the Criterion of Words' Written Forms" in 1962, and then it was published in the famous publication "Chinese". So far, the academic circle has been always study about heterographs. The concept of heterographs was first proposed by Gao Gengsheng, and it was defined in his article "On Sorting out Heterographs", which was published in "Chinese" in 1966. In 1985, Mr. Fu Yoghe wrote an article "About Heterographs' Criterion", which was published in Chinese. The concept of variant-form word was put forward. From then on, the two concepts both referred to the same meaning.

Although the definitions of heterographs are slightly different meaning, both of them have the feature of the different shape but same meaning terms. For instance, Gan Gengsheng (2000) mentioned that heterographs mean synonyms, and homonyms but different forms, and they are frequently used in society life and they can be taken

\footnotetext{
* Acknowledgements: Thanks for The fund supported by Jilin Provincial Education Department (No. 2015. 17, The 12th Five-Year of Social Science Research). JIN Ya-shu, M.A., Associate Professor, College of Humanities and Social Science, Yanbian University, Yanji, China.
} 
place in dictionary. Su Xinchun (2002) has an idea that heterographs are the words which are the same in sound and meaning, but different in form. There exists some slight different opinions between "the same sound" and "the similar sound". Yangchun (2004) believes that the heterographs refer to the different forms of one word in a literal way. Specifically speaking, these written forms coexist in different forms, the same sound, the same meaning and they are monosemantic and in common use. Pan Jinghan (1994) refers that the standard to recognize heterographs is that the completely same word meaning and usage and the same or similar sound (including ancient sound and dialectal accent), and the replaceability at all contexts. Some opinions are still not completely unanimous, but the main view has been close to accordance.

In Gao Gengsheng's article "On Sorting out Heterographs Again", heterographs was described as "Heterographs refers to the words which are in the same sound, the same meaning and in different forms, and which are coexisting and co-used in society. These conditions should exist at the same time" (p. 7). The basic characteristics of heterographs were summarized by this definition and the standard was clear, so that it was accepted by most people.

\section{The Identification of the Heterographs in Northeast Dialect}

In this study, we agree that Mr. Gao's view on heterographs in mandarin. However, the same sound should not be the complete standard of heterographs in Northeast dialect, because the word's phonetic forms revealed the historical development of different periods and different areas in sound. We advocate that the completely same word meaning and usage can be taken place in all contexts and all of the words, which is in the same or similar sound, and can be defined as dialects heterographs. Therefore, we identify the heterographs in Northeast dialect as the words which are in the same sound, the same meaning and in different form, and which are coexisting and co-used in the language system of Northeast dialect.

"Different forms" refers to the different written styles of one word in a literal way. "Coexisting and co-used in the language system of Northeast dialect" means that the heterographs which need criterion is a kind of language phenomenon, which refers to those heterographs currently used in modern Northeast dialect.

"The same sound" requires that the sound of initial consonant and simple vowel should be exactly the same. There are three conditions: (1) The sound of initial consonant and simple vowel are exactly the same; (2) They are not the same originally, but when rhotic accent is added into the whole accent, they are the same; (3) Not the same originally, when read in a soft voice, they are the same. Avoiding complicating the issues, some words which need criterion can be described as synonym. Loanwords heterographs are sorted out as a kind of special heterographs.

"Similar sound" means the similar sound of initial consonant and simple vowel. According to the features of Northeast dialect, there are four conditions: (1) The same initial consonant and similar simple vowel; (2) The similar initial consonant and same simple vowel; (3) The similar affixes; (4) The similar tone pitch.

"The same meaning" means that, strictly speaking, the conceptual meaning, emotional meaning and grammatical meaning are exactly the same and they can replace each other in any contexts.

It is one of difficulties of defining scope of heterographs is to discriminate the boundary between heterographs and homophone-synonyms. On the article of Liu Yonggeng (1990), "Some Issues on Heterographs", he advercates that "For a sort of words in the same sound and meaning but in different writing 
styles, the key to discriminate them as heterographs or homophone-synonyms is to inspect that whether they are exactly equal on meaning or just similar". If they have even slight differences on lexical meaning (including conceptual meaning, emotional meaning, stylistic meaning and imaginal meaning), grammatical function (including the characteristic or property of a certain word and syntactic features) and application, they can be regarded as homophone-synonyms, because they have different values in communication. Although there are clear standard on theory, there are still some differences in usage and language sense in reality. There are still some disagreements on some specific words. A lot of meanings and usages are still in development for many words. We need to have an motivational analysis on this kind of words with profound study.

In terms of the exact same meaning, the exact heterographs should be the same on separation of meanings. However, there are two situations which are worth discussing. One is that there is the same separation of meaning for two writing styles, while one of them has another. In mandarin, this kind of situation is classified in heterographs in majority, and difined as subsumed heterographs like subsumed variant Chinese character. Another situation is that there is the same separation of meaning for two writing styles, while both of them have their owns. They cannot be replaced by each other. Some people believe that they are heterographs and called crossed heterographs in the study of Mandarin heterographs. We think that on the point that the same separation of meaning can be replaced, they are similar with heterographs. But in terms of word criterion, it is just a kind of minor adjustment for separation of meaning, not on the issue of acceptance or abandon of the form of word. It is not only different with classical heterographs (completely the same), but also different with subsumed heterographs. The criterion of classical heterographs is totally about the accept of the form (In general, the form with more separation of meaning would be accepted. The less would be abandoned.). Although some of the criterion of subsumed heterographs is to be or not to be, most of them are adjustments on separation of meanings (Implement differentiation).

Because the research of dialect is in virgin state and we haven't any references on heterographs in Northeast dialect, the study of this time is just an initiation. Therefore, the collection and arrangement of Northeast dialect are very cautious. If necessary, some annotation will be added to defined the scope in order to avoid misunderstanding and confusion. So-called subsumed heterographs and crossed heterographs are more complex in Northeast dialect, so they will not be collected in the arrangement.

\section{The Reason That the Heterographs in Northeast Dialect Come Into Being}

The condition of the the heterographs in Northeast dialect is complex. Apart from the language itself, there are a variety of factors, including immigration and linguistic integration.

\section{The Unique Phonetic Feature of Northeast Dialect}

The feature of initial consonant: The vague distinguish between level-lingual and cacuminal, always read "z $\backslash \mathrm{c} \backslash \mathrm{s}$ " as "zh $\backslash \mathrm{ch} \backslash \mathrm{sh}$ ", and read "zh $\backslash \mathrm{ch} \backslash \mathrm{sh}$ " as "zlcls"; Adding [n] at zero-initial-syllable, for example, always add [n] at "ai 、ao、ou、an、en、 ang"; Mixing [n] and [1], for example, read [nən] as [lan].

The feature of simple vowel: Add $[ə]$ at $[\mathrm{o}]$ and the combination of $[\mathrm{p}],\left[\mathrm{p}^{\mathrm{c}}\right],[\mathrm{m}],[\mathrm{f}]$; Add $[\eta]$ at some spoken words. There are a lot of retroflexed suffixation sound and toneless sound in tone. The feature on tone is an important reason to come into being the heterographs in Northeast dialect. 


\section{The Integration of Multi-ethnic, Multi-language and Multi-culture}

The Northeast dialect was formed only two or three hundred years ago. Due to the mutual contact among different ethics for a long time, languages acquired mutual absorption. From the forming process of Northeast dialect, in the period of Liao, Jin and Yuan dynasties, the source of Han immigrant population is complex. At this time, none of language played a leading role. It's the primary stage of Northeast dialect to come into being. In the period of Ming and Qing Dynasty, it's the stage of coming into being and developing. A majority of features of Northeast dialect have come into being and connected with Beijing dialect. At this stage, based on Beijing dialect, Northeast dialect has come into being after integrating some components from other dialects. The late Qing Dynasty is an important period of the lexical system construction of Northeast dialect. In the lexical system, Youyan dialect remains dominant. At the same time, it also absorbed in the minority language of the native residents in northeast, dialects from other provinces and loanwords. In the end of the Qing dynasty, with the declination of Manchu language in northeast area and the transition from using Manchu language to using Chinese, Youyan dialect advocated its dominant position. However, Manchu language didn't disappear, instead, it stayed in the lexical system of Northeast dialect as substrate words with other minority languages in northeast. The late Qing Dynasty is the period with the largest immigrant population at home and abroad. Therefore, dialects from other provinces such as Shandong province, and loanwords rapidly added into the lexical system of Northeast dialect, which is a unique language contact phenomena. When dealing with these language, it is inevitable that the heterographs come into being.

\section{Type Analysis of Heterographs in Northeast Dialect}

It's still a virgin stage on the study of heterographs in dialect, no matter in the study of dialect or lexicology. Taking the heterographs in Mandarin as the reference, we have summed up and sorted out the information of heterographs in Northeast dialect for analysis.

We divided Northeast dialect into homophonies, allophones and Yixu Heterographs, which are in different order in it. The former two were summarized based on the previous discussion on the concept of heterographs in Mandarin, including a part of loadwords. Homophonies refer to the heterographs in the same sound and meaning but in different forms. Allophones refer to the heterographs in the similar sound but in different forms, divided into three condition, including with similar initial consonants, with similar simple vowels and with similar tone pitches. Yixu Heterographs is a kind of special phenomenon. Several groups of heterographs in Northeast dialect belong to the words with the same morpheme.

Taking Northeast dialect dictionary written by Mr. Ma Sizhou and Mr. Jiang Guanghui as the main language material, we sorted out 17 groups of homophonies, 3 groups of Yixu Heterographs and 113 groups of allophones.

One of the reasons to come into being allophones in Chinese is the vast territory and various dialects in northeast of China. Allophones which were made by people from different areas promoted the changes of Chinese characters, so that the allophones were influence by different dialects. Therefore, dialects would produce allophones.

Among these heterographs, one is produced with different understanding of the meaning so that it leads to the difference in forms. This kind of heterographs has the feature of motivations when forming. They focus on the distinguishment of meaning. Based on their own meaning, they are able to express the same meaning from 
different aspects. The other is led to the arbitrariness. Most of them are loanwords which are transliterations or some onomatopoeia. Due to the large number of homophones in Chinese, it is certain of arbitrary in homophones.

\section{Conclusion}

It is natural to come into being heterographs, but it is not reasonable. In fact, it increases the burden of learners and users. There is significant immigrant cultural features in the history of northeast. When communicating with Northeast dialect, some other dialects from other provinces and loanwords have been absorbed in Northeast dialect and become the important part of the lexical system of Northeast dialect, enriching its locality and uniqueness. These integrations display that the lexical system of Northeast dialect is open and dynamic. Owing to the influence from this situation, it is inevitable to come into being heterographs.

On selecting of heterographs in Northeast dialect, people prefer to use the forms which are able to display the Motivational meaning, Denotative meaning and Psychological meaning. Therefore, most of them are produced through the adjustment of usage between form and meaning, so that we should be positive and cautious when using the heterographs in Northeast dialect.

\section{References}

FU, Y. H. (1985). Questions about the normalization of heterographs. The Reforms of Chinese Characters, 1, 21-23.

GAO, J. S. (1991). About the normalization of heterographs. Language planning, 1, 65-69.

GAO, J. S. (1993). About the normalization of heterographs again. Language planning, 6, 7-11.

HOU, M. (1992). Questions of the normalization of heterographs. Language planning, 3, 19-22.

LIU, J. S. (1996). The principles of normalization about the heterographs. Language planning, 4, 8-9.

MA, S. Z. (1991). Jiang guanghui. Dictionary of Northeast dialect.

TIAN, Z. Y. (1993). Questions about the normalization of heterographs in morden Chinese. Journal of Jinzhou Teachers College, (1), 25-32.

XU, H. G., \& ZHANG, D. M. (1988). Concise dictionary of the Northeast dialect. Changchun city: Jilin literary and history Press. 